\title{
Formation of negatively charged Agl colloid nanoparticles by condensation
}

\author{
Daina Kalnina ${ }^{1, a^{*}}$, Karlis Agris Gross ${ }^{1, b}$, Arturs Medvids ${ }^{2, c}$ \\ and Pavels Onufrijevs ${ }^{2, d}$ \\ ${ }^{1}$ Biomaterials research laboratory, Riga Technical University, Latvia \\ ${ }^{2}$ Institute of Technical Physics, Riga Technical University, Latvia \\ adaina.kalnina@rtu.lv, bkgross@rtu.lv, 'cmedvids@latnet.lv, donufrijevs@latnet.Iv
}

Keywords: silver iodide colloids, UV absorption spectra, stability, $\zeta$-potential

\begin{abstract}
The stability of silver halide colloids is reported to be important for the toxicological outcome. This study shows a well-suited and cheap condensation reaction to obtain negatively charged silver iodide (AgI) nanoparticles without additional stabilization agents. Charged AgI colloids were synthesized from silver nitrate and potassium iodide solutions. An excess of potassium iodide not only imparted a negative charge, but provided a narrow particle size distribution $(50 \pm 10 \mathrm{~nm})$. The change of optical properties in the colloid was investigated by UVVIS spectroscopy. A silver iodide exciton absorption band at peak $\sim 421 \mathrm{~nm}(2.93 \mathrm{eV})$, red-shifted over time. The peak at half maximum intensity increased from $13.3 \mathrm{~nm}$ to $14.8 \mathrm{~nm}$, characterizing the dispersity of AgI colloidal particles. Colloidal particles stabilized after 33 hours. In-situ realtime UV-VIS measurements provide a tool to adjust the particle characteristics and may serve to further optimize the performance in biological applications.
\end{abstract}

\section{Introduction}

Silver iodide colloids continue to change after synthesis and offer a yet unexplored avenue for adjusting nanoparticle characteristics. Silver and silver halides find a wide range of applications. Silver nanoparticles are attractive for their photosensitivity, ionic conductivity and antibacterial behaviour. Silver iodide - an ionic semiconductor with a $\sim 3.0 \mathrm{eV}$ band gap [1] - finds application as a catalyst in organic reactions [2], shows promise as an anticancer and X-ray contrast agent [3]. This work investigates changes of negatively charged silver iodide colloids over time by detecting variations in the optical characteristics.

The stability and aging of silver nanoparticles has been reported to be important for reducing toxicity [4]; correlated with the release of $\mathrm{Ag}^{+}$ions. Silver is chemically stable, and not toxic [5], but there is some concern when silver is present as a cation. Ultimately, the synthesis method and the presence of residual contaminants (e.g., $\mathrm{Ag}^{+}$, residual reducing and stabilizing agents) could also account for the observed toxicity $[6,7,8]$. This will influence the properties even when incorporated into a resorbable matrix, such as cements [9]. In addition to solubility, particle charge plays another characteristic that influences the toxicity in water [10].

To improve nanoparticle stability, stabilizing agents are typically introduced during synthesis [11], but another approach would be to stop processing at the required time to capture the required properties. More and more attention is given to syntheses of silver containing nanoparticles and so a detailed description needs to be provided on the synthesis route, aging, stabilisation and cleaning $[12,13]$. Nanoparticle purity is becoming increasingly important and is receiving increased attention by regulatory committees [14]. Different mechanisms have been proposed for determining the purity: double-layer compression, charge neutralization, entrapment during precipitation [15]. Colloidal particle stability varies over time and this needs attention to provide reproducible pure nanoparticles. Iodide is chosen both to impart a negative charge and stabilize nanoparticles. The objective here is to show the formation of a stable $\mathrm{AgI}$ colloid in an aqueous solution of $\mathrm{AgNO}_{3}$ and $\mathrm{KI}$ and to determine changes over time. 


\section{Materials and Methods}

Colloids of silver iodides were synthesized using a condensation reaction route from silver nitrate and potassium iodide solutions. Colloid charge was imparted from an excess of potassium iodide in solution. A $0.01 \mathrm{M} \mathrm{AgNO}_{3}$ solution was added drop-wise to a rapidly stirred $0.01 \mathrm{M} \mathrm{KI}$ solution. The larger iodide to silver ratio $(1.0,1.8,2.0$ and 4.5) ensured the removed of free silver ions from solution. As AgI is light sensitive, all glassware was enclosed in aluminium foil to keep the solution and powder in darkness.

Absorption spectra of AgI nanoparticles were obtained using a Shimadzu UV spectrometer (300$500 \mathrm{~nm}$ range, $0.1 \mathrm{~nm}$ interval, every 30 minutes). Particle shape and size was determined by field emission scanning electron microscopy (SU70, Hitachi). Zeta potential of the obtained dispersions was measured on a Zetasizer Nano ZS analyser with an integrated $4 \mathrm{~mW} H e-N e$ laser, $\lambda=633 \mathrm{~nm}$ (Malvern Instruments Ltd.).

\section{Results and Discussion}

The AgI condensed from equimolar amounts of silver and iodide are bulk crystals (Fig.1). Therefore, it is not possible to measure the zeta potential (shown as $\zeta$-potential in Table 1). Greater concentrations of iodide produced the charged colloid, by imparting a charge to the particle surface that kept the particles dispersed within the solution.

Table 1. Characteristics of silver iodide sols for increasing concentrations of iodide.

\begin{tabular}{|c|c|c|c|c|c|c|}
\hline \multirow{2}{*}{$\begin{array}{c}\text { Sample } \\
\text { nr. }\end{array}$} & \multirow{2}{*}[\mathrm{I}^{-}]{$/\left[\mathrm{Ag}^{+}\right]$} & \multicolumn{2}{|c|}{$\zeta$ - potential, $\mathrm{mV}$} & \multicolumn{2}{|c|}{$\lambda_{\max }, \mathrm{nm}$} & \multirow{2}{*}{$\Delta \lambda_{\max }, \mathrm{nm}$} \\
\cline { 3 - 6 } & & $(\tau=0.5 \mathrm{~h})$ & $(\tau=24 \mathrm{~h})$ & $(\tau=0.5 \mathrm{~h})$ & $(\tau=24 \mathrm{~h})$ & \\
\hline 1. & 1.0 & - & - & - & - & \\
\hline 2. & 1.8 & 48 & 59 & 427 & 429 & 2 \\
\hline 3. & 2.0 & 45 & 54 & 429 & 431 & 2 \\
\hline 4. & 4.5 & 54 & 56 & 421 & 425 & 4 \\
\hline
\end{tabular}

High $\zeta$-potential $(>45 \mathrm{mV})$ indicates a repulsive force that prevents particle aggregation and supports nanoparticle stability (attributed to $\left[\mathrm{I}^{-}\right]_{0} /\left[\mathrm{Ag}^{+}\right]_{0}$ and ionic strength). Electrostatic stabilization from the electrical double layer arises from adsorbed ions [15].

Electron microscopy showed different particle characteristics for the dried AgI. The condensation reaction with $\left[\mathrm{I}^{-}\right]_{0} /\left[\mathrm{Ag}^{+}\right]_{0}=1$ showed irregular, large AgI particles with a large size distribution $(0.2$ $2.0 \mu \mathrm{m})$, but an excess of iodide produced rounded equisized AgI nanoparticles (50 $\pm 10 \mathrm{~nm})$, Fig. 1. Other work has shown that an excess of iodide stabilizes the $\beta$-AgI phase [16].
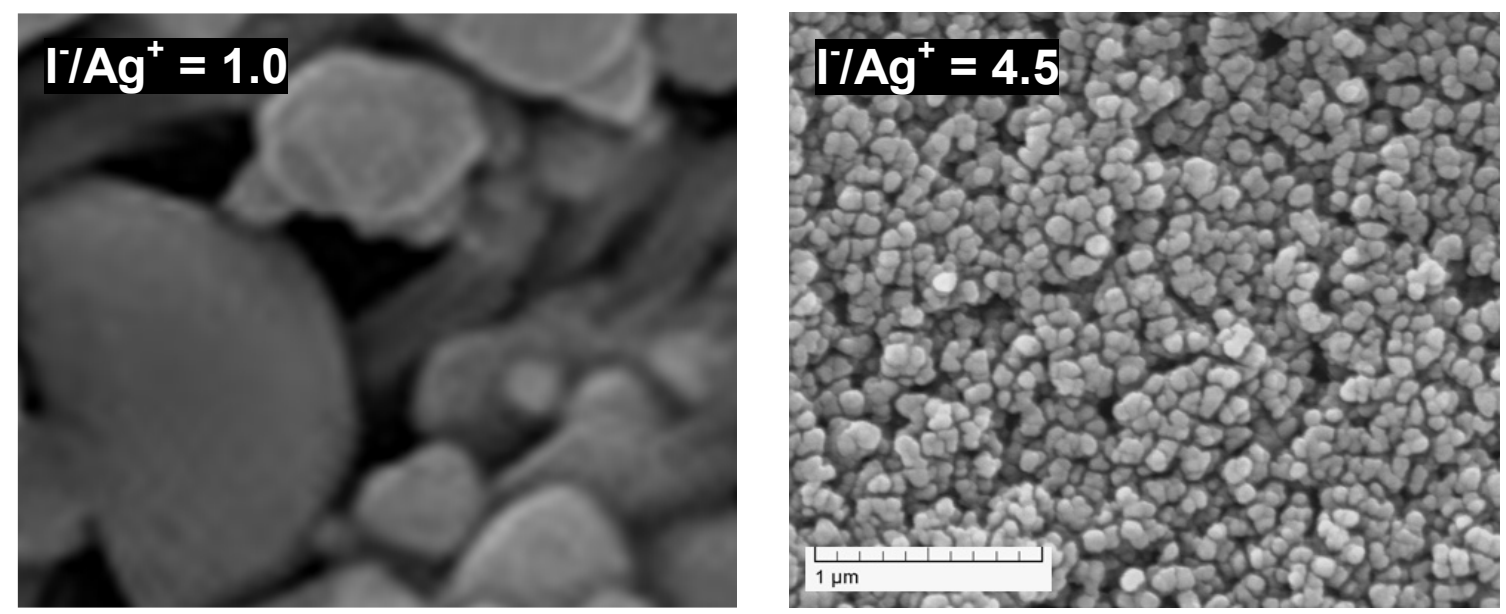

Fig.1. Large angular dried AgI particles obtained from an equimolar iodide and silver concentration, and rounded, equisized dried particles from an excess of iodide. 
An increase of AgI colloid optical absorption occurred over time, Fig. 2. A peak at $\lambda=425 \mathrm{~nm}$ and a shoulder at $\lambda=330 \mathrm{~nm}$ suggests the presence of excitons in AgI [17]. The exciton peak position (curve 1, Fig.3) and its intensity (curve 2, Fig.3) changed over time (synthesis nr. 4) causing a red peak shift of $27,6 \mathrm{meV}$ at $\max \lambda=425 \mathrm{~nm}$ and $20,75 \mathrm{meV}$ at $\lambda=330 \mathrm{~nm}$, Fig. 3. The increase in peak width at half maximum (FWHM) from $13.3 \mathrm{~nm}$ to $14.8 \mathrm{~nm}$ reflected an increased dispersity of AgI colloidal particles.

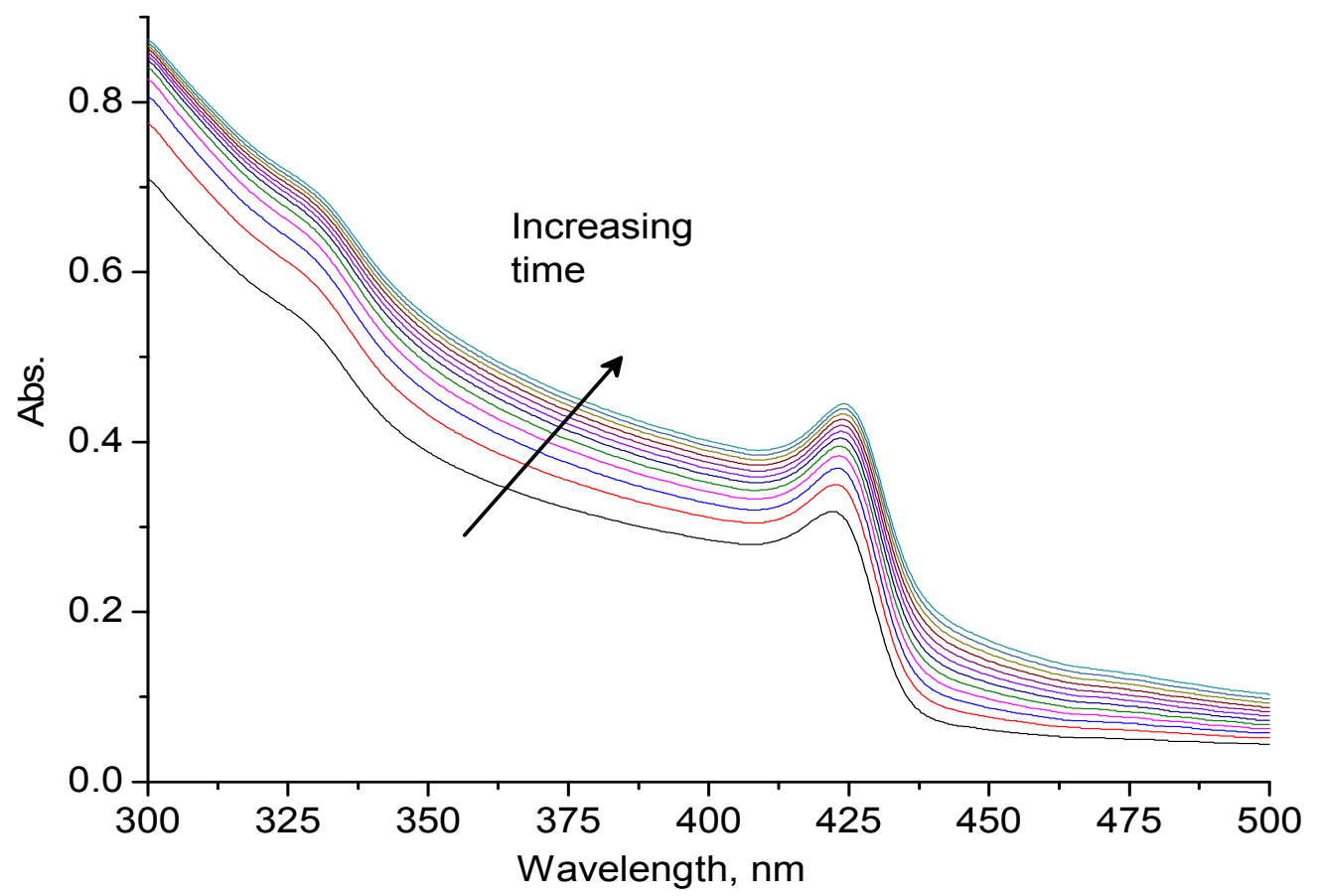

Fig. 2. UV-VIS time dependent AgI colloid absorption spectra recorded over time showing a "red shift".

A red shift in the electromagnetic wave absorbance may be related to the particle size, shape, aggregation state or dielectric constant of the surrounding medium [18]. The AgI colloid is stabilized in solution after 33 hours.

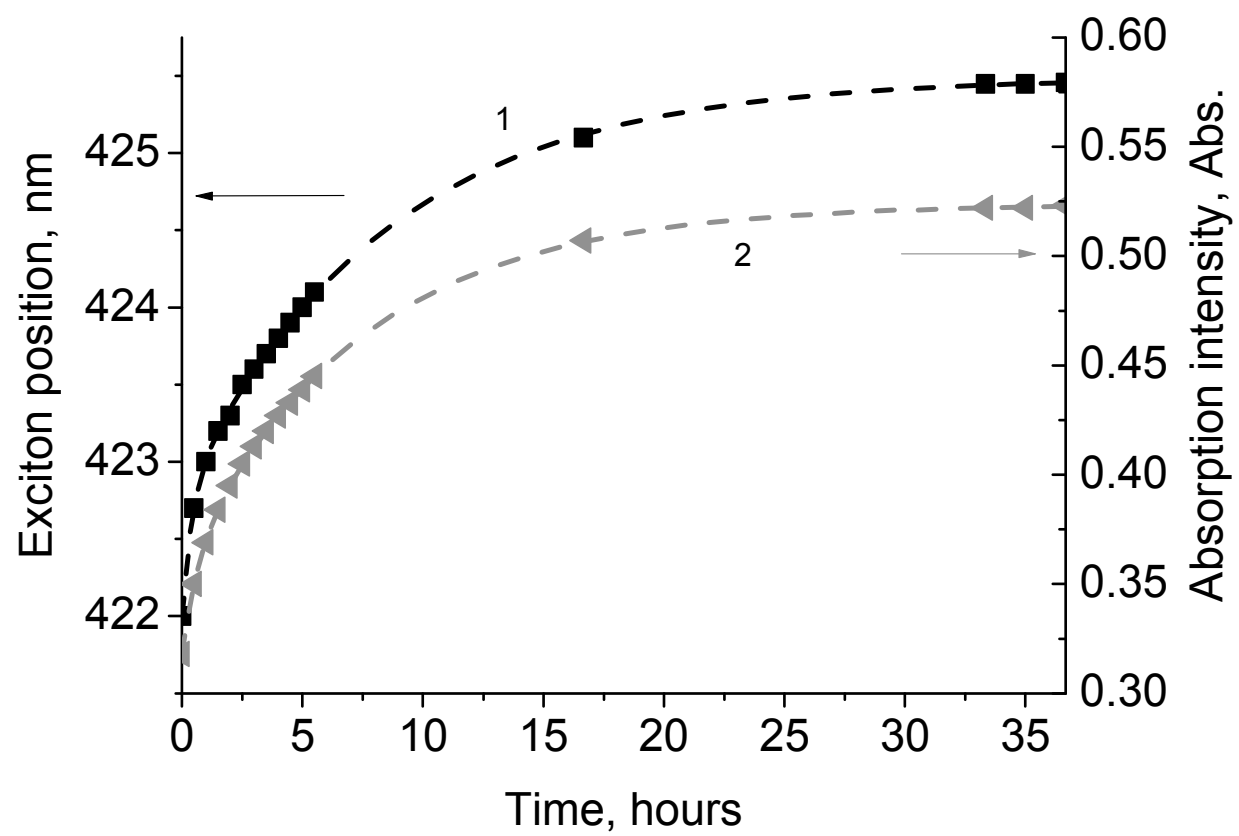

Fig.3. Time-dependent change of exciton absorption band position and intensity. 
In-situ experiments may also reveal a change in charge over time during synthesis. Such experiments would require the combination of different characterization tools during synthesis. This will help understand a more complete change in particle characteristics and influence on properties.

\section{Summary}

An excess of potassium iodide produced a charged and stable colloid that upon drying revealed 50 $\pm 10 \mathrm{~nm}$ sized nanoparticles. UV absorption by AgI nanoparticles showed a continual change over time that stabilized at 33 hours. UV adsorption spectra measurements give an opportunity to follow colloid stabilization over time. This study has shown that the condensation reaction is a cheap alternative to obtain negatively charged silver iodide colloidal particles without additional stabilization agents.

\section{References}

[1] Y. Wang, J.Mo, W.Cai, L.Yao, L.Zhang, Large-scale synthesis of B- AgI nanocrystals, Materials Letters, 56 (2002) 502-506.

[2] J. Safaei-Ghomi, M.A. Ghasemzadeh, Silver iodide nanoparticle as an efficient and reusable catalyst for the one-pot synthesis of benzofurans under aqueous conditions, J. Chem. Sci. 125, (2013) 1003-1008.

[3] Y. Sakurai, H.Tada, K. Gonda et al., Development of silica-coated iodide nanoparticles and their biodistribution, J. Exp. Med. 228 (2012) 317-323.

[4] S. Kittler, C. Greulich, J. Diendorf et al, Toxicity of silver nanoparticles increases during storage because of slow dissolution under release of silver ions. Chem. Mat. 22 (2010) 4548-54

[5] A.B.G. Lansdown, A Pharmacological and toxicological profile of silver as an antimicrobial agent in medical devices. Advances in Pharmacological Sciences, (2010) Article ID 910686,16 pages.

[6] M.E. Samberg, P.E. Orndorff, N.A. Monteiro-Riviere, Antibacterial efficacy of silver nanoparticles of different sizes, surface conditions and synthesis methods. Nanotoxicology 5 (2011) 244-253.

[7] E. Navarro, F. Piccapietra, B. Wagner et al., Toxicity of silver nanoparticles to Chlamydomonas reinhardtii, Environ. Sci. Technol. 42 (2008) 8959-8964.

[8] T.M. Tolaymat, A.M.E Badawy, A. Genaidy et al., An evidence-based environmental perspective of manufactured silver nanoparticle in syntheses and applications: A systematic review and appraisal of peer-reviewed scientific papers, Sci. Total Environ. 408 (2010) 999- 1006.

[9] S. Jacquart, R. Siadous, C. Henocq-Pigasse, et al, Composition and properties of silvercontaining calcium carbonate-calcium phosphate bone cement, J. Mater Sci: Mater Med. (2013)

[10] A.M.El Badawy, R.G. Silva, B. Morris, et al., Surface charge dependent toxicity of silver nanoparticles. Environ. Sci. Technol. 45 (2011) 283-287.

[11] P. White, J. Hjortkjaer, Preparation and characterisation of a stable silver colloid for SER(R)S spectroscopy, Raman Spectrosc. 45 (2014) 32-41.

[12] B. Reidy, A. Haase, A. Luch, K.A. Dawson, I. Lunch, Mechanisms of silver nanoparticle release, transformation and toxicity: A critical review of current knowledge and recommendations for future studies and applications, Materials. 6 (2013) 2295-2350.

[13] M. Tourbin, A. Al-Kattan, C. Drouet, Study on the stability of suspensions based on biomimetic apatite's aimed at biomedical applications. Powder Technology. 255 (2014) 17-22. 
[14] Al-Kattan, P. Dufour, C. Drouet, Purification of biomimetic apatite-based hybrid colloids intended for biomedical applications: a dialysis study, Colloids and Surfaces B Biointerfaces. 82 (2011) 378-384.

[15] J. Dutta, H. Hofmann, Self -organization of colloidal nanoparticles, in: H.S. Nalwa (Ed.), Encyclopaedia of Nanoscience and Nanotechnology, American Scientific Publisher, CA, (2004) 617-640.

[16] Vukić, Marija R.; Veselinović, Dragan S.; Marković, Vesna G, Crystalline forms of silver iodide II. Determination of phase transformations, Journal of the Serbian Chemical Society. 72 (2007) 857

[17] D. Bharathi Mohan and C. S. Sunandana, Iodization of rf sputter induced disordered Ag thin films reveals volume plasmon-exciton "transition", Journal of Applied Physics. 100 (2006), 064314-1-10

[18] T.C. Prathna, N. Chandrasekaran, A.M. Raichur, A. Mukherjee, Biomimetic synthesis of silver nanoparticles by Citrus limon (lemon) aqueous extract and theoretical prediction of particle size. Colloids and Surfaces B: Biointerfaces. 82 (2011) 152- 159. 